\title{
Reporter Gene Assays
}

Running head: Reporter gene assays

Andy M.F. Liu M.Sc., David C. New Ph.D., Rico K.H. Lo M.Phil and Yung H. Wong Ph.D. ${ }^{\dagger}$

Department of Biochemistry, the Molecular Neuroscience Center, and the Biotechnology Research Institute, Hong Kong University of Science and Technology, Clearwater Bay, Hong Kong, China.

${ }^{\dagger}$ Address correspondence and reprint requests to Prof. Y.H. Wong, Department of Biochemistry, Hong Kong University of Science and Technology, Clearwater Bay, Hong Kong, China. E-mail: boyung@ust.hk, Tel: 2358 7328, Fax: 23581552. 


\begin{abstract}
Reporter gene assays are versatile and sensitive methods of assaying numerous targets in high-throughput drug screening programs. A variety of reporter genes allow users a choice of signal that can be tailored to the required sensitivity, the available detection apparatus, the cellular system employed and the compatibility with multiplexed assays. Promoters used to drive the reporter gene expression can either be activated by a broad range of biochemical pathways or by the selective activation of individual targets. In this chapter, we will introduce some of the considerations behind the choice of reporter gene assays and describe the methods that we have used to establish 96-well format luciferase and aequorin assays for the screening of ligands for $G$ protein-coupled receptors.
\end{abstract}

Key Words: Reporter gene, luciferase, aequorin, GPCR, receptor, G protein, chimera, STAT, CRE, NFkB.

\title{
1. Introduction
}

The development of cell based high-throughput screening platforms owes its success to our ability to manipulate the genetic and biochemical composition of eukaryotic cells. Notably, cells can be engineered to express specific gene products in response to a given stimulus. The gene product itself may posses an inherent property that enables it to be directly measured, e.g. green fluorescent protein (GFP), or it may display enzymatic activity that can be monitored, e.g. luciferase. Alternatively, the gene product may respond to changes in the levels of a signalling molecule, e.g. the $\mathrm{Ca}^{2+}$ ion 
activation of aequorin luminescence. The continuing development of genetically engineered vectors coupled with powerful detection techniques allows many cell types to be engineered as biodetectors for a myriad of classes of biochemicals and signalling pathways.

The reporter gene unit consists of a promoter and the reporter gene. When establishing an assay system a number of factors need to be considered in order to optimize the assay. The choice of promoter, the number of copies of the promoter and the nature of the reporter gene will allow control of the basal level of reporter gene activity, control of the specificity of activation and control of the degree of stimulation measured [1]. Endogenous promoters, such as c-fos, the cAMP response element (CRE) or the estrogen response element are commonly used but may suffer interference from endogenous intracellular signalling events. However, their regulation by multiple signalling pathways makes them widely applicable, even allowing drugs that activate different signalling events to be identified in the same assay format. Alternatively, exogenous promoters such as the yeast Ga14 response element system can be used to reduce unwanted activation by native transcription factors [2]. There are many examples of the optimization and employment of a variety of promoters in high-throughput screening programs [e.g. 3].

The reporter gene itself should ultimately generate a signal that can be clearly identified. The reporter gene products can either be intracellular or extracellular in nature. Intracellular products are retained in the cell for quantification in situ or following cell lysis. Extracellular products are secreted into the extracellular medium for assay, allowing for repeated experimentation and sampling without disturbing the cells. 
Commonly used intracellular reporter genes are chloramphenicol acetyltransferase (CAT), $\beta$-galactosidase, luciferase, aequorin and GFP. Extracellular reporter genes are usually secreted placental alkaline phosphatase (SPAP) or $\beta$-lactamase [3 and references therein]. Each reporter gene has its advantages and disadvantages in terms of sensitivity, time taken for signal generation, ease of signal detection (e.g. products can be radioactive, fluorescent, bioluminescent etc), cost, miniaturization and automation. A further consideration may also be the compatibility within dual reporter gene assays or multiplexed assays.

A recent report of a dual reporter gene assay demonstrated the ease with which signals from two different classes of G protein-coupled receptors (GPCRs) can be measured simultaneously. Two cell lines were generated that expressed either CRE coupled to firefly luciferase (CRE-luc) or a 12-O-tetradecanoyl-phorbol-13-acetate (TPA)-response element coupled to Renilla luciferase. Coseeding of the reporter cell lines allowed independent or simultaneous measurement of ligand activation of GPCRs that activate distinct intracellular signalling pathways [4]. The authors have also suggested that the inclusion of a third GFP-based reporter construct would allow 3 separate GPCRs to be co-assayed. Reporter gene assays have also proven amenable to inclusion in multiplexed assays that also measure $\mathrm{Ca}^{2+}$ mobilization in a 384-well format. For example, by using the $\beta$-lactamase based GeneBLAzer ${ }^{\circledR}$ reporter gene system (Invitrogen Corporation, Madison, WI) and loading the same cells with the calcium sensing Fluo-4 NW dye, researchers were able to rapidly verify positive hits in a drug screen by correlating the data from $\mathrm{Ca}^{2+}$-based assays and reporter gene assays performed sequentially on the same cells [5]. Running these two assays also facilitated the rapid 
elimination of false positives generated by either assay format, allowing huge savings in cost and time to be made.

In our experience, reporter gene based assays are an effective way for laboratories with limited resources to develop and deploy innovative and sensitive high-throughput screening programs. Reporter gene assays can be applied to a huge range of targets including GPCRs, nuclear receptors, receptor tyrosine kinases, enzymes and transcription factors. Furthermore, by tailoring the incorporation of relevant promoters and reporter genes into different assays, diverse screening programs can be established that utilize common detection equipment, increasing the cost efficiency of drug screening.

We use both luciferase and aequorin reporter genes. The luciferase genes are driven by STAT3, NFkB or CRE promoters. These allow us to assay most GPCRs, with

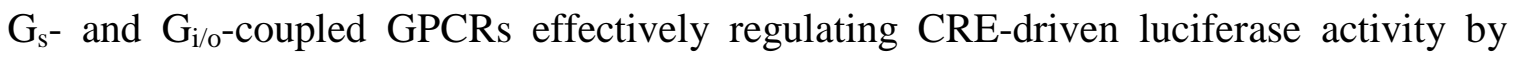
modulating intracellular cAMP levels [6]. $\mathrm{G}_{\mathrm{q}^{-}}$and $\mathrm{G}_{\mathrm{i} / 0^{-}}$-coupled GPCRs are assayed by either STAT3- or NFkB-driven assays while $\mathrm{G}_{12 / 13}$-coupled receptors generally promote NFkB-driven luciferase activity [7-9]. The flexibility of all of our reporter gene assays is greatly enhanced by the incorporation of promiscuous or chimeric $G$ protein $\alpha$-subunits, notably $G \alpha_{16}, 16 z 25$ or $16 z 44$ [10]. These allow virtually all GPCRs tested to date to promote the activation of phospholipase $\mathrm{C} \beta$ ( $\mathrm{PLC} \beta) /$ inositol trisphosphate $\left(\mathrm{IP}_{3}\right) / \mathrm{Ca}^{2+}$ pathways, enabling a single assay to be used to screen for GPCR activity. Numerous chimeras have been stably or transiently expressed in a variety of cell lines and incorporated into a number of other assay platforms, including FLIPR, microarrayed compound screening and yeast autocrine selelction [11,12]. In fact, cell lines stably expressing GPCRs and promiscuous G proteins are commercially available (Chemicon 
Int., California, USA). Using endogenous and chimeric G proteins we, and others, have successfully incorporated dozens of GPCRs into aequorin-based reporter gene screening programs [13-15]. In this chapter we will detail the methods that we regularly use in our own GPCR analysis and screening programs. 


\section{Materials}

1. Dulbecco's Modified Eagle’s Medium (DMEM) (Gibco, Bethesda, MD).

2. Modified Eagle’s Medium (MEM) (Gibco).

3. OPTI-Modified Eagle’s Medium (Opti-MEM, Gibco).

4. Phenol red-free DMEM (Sigma-Aldrich).

5. Fetal bovine serum (FBS, Gibco).

6. Dulbecco’s Phosphate-buffered saline (PBS, Gibco).

7. Hanks' Balanced Salt Solution (HBSS) with or without calcium chloride (Gibco).

8. Solution of trypsin (1X) is diluted from trypsin (10X) with PBS (Gibco).

9. Solution of Penicillin/Streptomycin (20X) (Gibco).

10. Zeocin (250 mg/ml) (Gibco).

11. Geneticin (G418) is dissolved in Opti-MEM at $100 \mathrm{mg} / \mathrm{ml}$ and stored at $-20^{\circ} \mathrm{C}$ (Calbiochem, San Diego, CA). The working concentration is at $50 \mathrm{mg} / \mathrm{ml}$.

12. Lipofectamine PLUS and Lipofectamine 2000 reagents (Gibco).

13. 2 X HEPES Buffer Saline (HBS) is made up of $280 \mathrm{mM} \mathrm{NaCl}, 10 \mathrm{mM} \mathrm{KCl}, 1.5$ $\mathrm{mM} \mathrm{Na} 2 \mathrm{HPO}_{4}, 12 \mathrm{mM}$ dextrose and $50 \mathrm{mM}$ HEPES (pH 7.05). The solution should be sterilized by passing through a $0.2 \mu \mathrm{m}$ filter.

14. Multichannel pipetman, Multipette Plus dispenser and Combitips Plus (5 $\mathrm{ml}$ and $2.5 \mathrm{ml}$ ) (Eppendorf, Hamburg, Germany).

15. Tissue-treated 96-well polystyrene white plates with flat bottoms (Nalge Nunc, Rochester, NY).

16. Polypropylene 96-well round-bottom block and solvent reservoir (USA Scientific, Ocala, FL). 
17. Conical 96-well polypropylene plates (Corning Costar).

18. Mitochondrial apoaequorin expression vector and coelenterzine f (Invitrogen, Bethesda, MD).

19. Luciferase reporter gene constructs with STAT3-, CREB- and NFkB-response elements (pSTAT3-Luc, pCRE-Luc and pNFkB-Luc) from Clontech (Mountain View, CA).

20. Luciferin and Luciferase lysis buffer are from a luciferase assay kit obtained from Roche (Mannheim, Germany).

21. 96-well format luminometer from EG\&G Berthold (Bundoora, Australia).

\section{Methods}

\subsection{Luciferase assay}

\subsubsection{Preparation of cells stably expressing CRE- or NFkB-driven luciferase} reporter genes

To minimize the variations in between different sets of experiments run on different days, stable cell lines carrying CRE- or NFkB-driven luciferase reporter genes have been generated. The plasmids carrying these reporter genes do not encode any selection markers. Therefore we cotransfect pcDNA3 to provide a means to select cells carrying both the reporter gene constructs and pcDNA3. We have found that it is not necessary to isolate a clonal cell line. Cells that survive the selection process are harvested and provide robust, consistent responses almost indefinitely. After the establishment of the stable cell lines, cDNAs encoding GPCRs and G proteins can be transiently introduced into the cells prior to the assays (section 3.1.2). 
1. 100-mm plates of $2 \times 10^{6}$ HEK 293 cells should be seeded in MEM medium containing 10\% FBS and 1X penicillin/streptomycin (see Note 1).

2. The following day the medium should be exchanged for $9 \mathrm{ml}$ of fresh MEM/10\% FBS/1X penicillin/streptomycin.

3. After three hours, prepare calcium phosphate transfection buffers as described below (the indicated volumes are required for each plate of cells):

a. Aliquot $500 \mu \mathrm{L} 2 \mathrm{X}$ HBS into a $5 \mathrm{~mL}$ sterile plastic tube.

b. Prepare the DNA mix in a second tube containing $50 \mu \mathrm{L}$ of $2.5 \mathrm{M} \mathrm{CaCl}_{2}$ (sterilized through a $0.2 \mu \mathrm{m}$ filter), $9 \mu \mathrm{g}$ of reporter gene cDNA, $1 \mu \mathrm{g}$ of selection marker DNA (pcDNA3) and make up volume to $500 \mu \mathrm{L}$ with $\mathrm{H}_{2} \mathrm{O}$.

c. Add DNA mix slowly to 2X HBS by pipetting dropwise.

d. Use a pipette to bubble air though the solution for $30 \mathrm{sec}$ to allow complete mixing.

e. Incubate at room temperature for $20 \mathrm{~min}$.

f. Add the mix to the cells dropwise with gentle swirling.

4. Incubate for $16 \mathrm{~h}, 37^{\circ} \mathrm{C}, 5 \% \mathrm{CO}_{2}$.

5. Aspirate the transfection medium, wash transfected cells with PBS three times to completely remove calcium phosphate-DNA precipitates and replenish with fresh MEM/10\% FBS/1X penicillin/streptomycin.

6. Incubate at $37^{\circ} \mathrm{C}, 5 \% \mathrm{CO}_{2}$ overnight.

7. Aliquot $50 \mu \mathrm{L}$ of $100 \mathrm{mg} / \mathrm{ml} \mathrm{G} 418$ to each $100 \mathrm{~mm}$ plate for selection tog give a final concentration of G418 of $500 \mu \mathrm{g} / \mathrm{ml}$. 
8. Fresh medium with $50 \mu \mathrm{L}$ of $100 \mathrm{mg} / \mathrm{ml} \mathrm{G} 418$ (to give a concentration of 500 $\mu \mathrm{g} / \mathrm{ml}$ ) is replenished every 3-4 days, after washing 3 times with PBS, until selection is finished (see Note 2).

9. Cells stably carrying luciferase reporter are maintained in $250 \mu \mathrm{g} / \mathrm{ml} \mathrm{G} 418$.

\subsubsection{Transient transfection of GPCR and G protein cDNAs into cell lines stably carrying luciferase reporter gene constructs (see Note 3).}

1. 15,000 HEK 293 cells carrying luciferase reporter genes (see section 3.1.1) are seeded per well of a 96-well white microplate using MEM (10\% FBS).

2. The following day, prepare the transfection cocktail as described below:

a. Mix $12.5 \mathrm{ng}$ GPCR cDNA and $37.5 \mathrm{ng}$ G protein cDNA per well.

b. Dilute $0.2 \mu \mathrm{L}$ of PLUS reagent with $25 \mu \mathrm{L}$ Opti-MEM per well.

c. Add PLUS/Opti-MEM mixture to the cDNA.

d. Incubate at room temperature for $15 \mathrm{~min}$.

e. Dilute $0.2 \mu \mathrm{L}$ of Lipofectamine reagent with $25 \mu \mathrm{L}$ Opti-MEM per well.

f. Add the diluted Lipofectamine to the cDNA/PLUS/Opti-MEM solution.

g. Incubate at room temperature for a further $15 \mathrm{~min}$.

3. Remove the growth medium from the white microplate seeded with reporter stable cell line.

4. Aliquot $50 \mu \mathrm{l}$ of the transfection cocktail to each well of the white microplate.

5. Incubate at $37^{\circ} \mathrm{C}$ for $3 \mathrm{~h}$.

6. Add $25 \mu \mathrm{L}$ of Opti-MEM containing 30\% FBS.

7. Further incubate at $37^{\circ} \mathrm{C}$ overnight. 
8. Proceed to section 3.1.4 for assay.

\subsubsection{Preparation of cells transiently expressing STAT3 promoter-driven luciferase reporter gene constructs (see Note 3).}

In contrast to the CRE promoter- and NFkB promoter driven luciferase reporter genes, attempts to establish HEK 293 cell lines stably expressing STAT3 promoterdriven luciferase reporter genes have resulted in luciferase signals that diminish with each passage of the cells. Therefore, transient introduction of the STAT3-driven luciferase reporter gene, along side the GPCR and G protein cDNAs, is employed.

1. 15,000 HEK 293 cells are seeded per well in a 96-well white microplate with MEM (10\% FBS).

2. The following day, prepare the transfection cocktail following step 2 of section 3.1.2, with a modification to step 2a, which should read;

a. Mix $10 \mathrm{ng}$ GPCR cDNA,10 ng G protein cDNA and $100 \mathrm{ng}$ STAT3 reporter cDNA per well.

3. Remove growth medium from the white microplate seeded with HEK 293 cells.

4. Distribute $50 \mu \mathrm{L}$ Opti-MEM to each well.

5. Aliquot $50 \mu \mathrm{l}$ of the transfection cocktail to each well.

6. Incubate at $37^{\circ} \mathrm{C}$ for $3 \mathrm{~h}$.

7. Add $25 \mu \mathrm{L}$ of Opti-MEM containing $30 \%$ FBS.

8. Further incubate at $37^{\circ} \mathrm{C}$ overnight.

9. Proceed to section 3.1.4 for assay. 


\subsubsection{Ligand induction}

\subsubsection{Ligand induction for NFKB- and STAT3-luciferase assays (see Note 4)}

1. Prepare and dilute appropriate agonists and/or antagonists using phenol red-free DMEM (50 $\mu \mathrm{l}$ will be required for each well of a 96-well plate).

2. Remove the transfection cocktail from the 96-well white microplate.

3. Aliquot $50 \mu \mathrm{l}$ of the prepared solutions with ligands or phenol red-free DMEM medium (as basal control) to appropriate wells. For determination of the contribution of $\mathrm{G} \alpha_{\mathrm{i} / 0}$ proteins to a response, wells should be pretreated with 100 $\mathrm{ng} / \mathrm{ml}$ PTX (in phenol red-free DMEM) for $4 \mathrm{~h}$ before ligand addition. The PTX can be added directly to the transfection cocktail.

4. Incubate at $37^{\circ} \mathrm{C}$ for $16 \mathrm{~h}$.

5. Remove medium completely.

6. Aliquot $25 \mu \mathrm{L}$ luciferase lysis buffer per well and store at $-80^{\circ} \mathrm{C}$ until use in the luciferase assay (see section 3.1.5).

\subsubsection{Agonist induction for CRE assays (see Note 4)}

1. Prepare diluted ligands and controls using phenol red-free DMEM.

2. Remove the transfection cocktail from the 96-well white microplate.

3. Aliquot $50 \mu \mathrm{l}$ of the prepared ligand solutions or controls, as appropriate, to the wells. Should forskolin be required, it should also be included at this point at a final concentration of $10 \mu \mathrm{M}$ (see Note 5).

4. Incubate for: 
a. To measure activation of adenylyl cyclase by $G_{s}$-coupled GPCRs: overnight at $37^{\circ} \mathrm{C}$.

b. To measure inhibition of adenylyl cyclase activity by $G_{i / 0}$-coupled receptors: $30 \mathrm{~min}$ at $37^{\circ} \mathrm{C}$. Remove stimuli, including forskolin, completely and wash twice with $100 \mu \mathrm{L}$ phenol red-free DMEM. Replenish with $50 \mu \mathrm{L}$ phenol red-free MEM and incubate at $37^{\circ} \mathrm{C}$ for 16 h.

5. Remove medium completely.

6. Aliquot $25 \mu \mathrm{L}$ luciferase lysis buffer per well and store at $-80^{\circ} \mathrm{C}$ until use in the luciferase assays (see section 3.1.5).

\subsubsection{Luciferase assay (see Note 6)}

1. Prepare luciferin solution and $1 \mathrm{X}$ luciferase lysis buffer and equilibrate both to room temperature ( $25 \mu \mathrm{l}$ of each solution will be required per well).

2. Prewarm the luminomoter to $25^{\circ} \mathrm{C}$. Ensure that the 96-well plates have been removed from $-80^{\circ} \mathrm{C}$ and thawed.

3. Charge injector P, of the 96-well luminometer (EG\&G Berthold, Bundoora, Australia), with luciferin solution and injector M with luciferase lysis buffer.

4. Inject $25 \mu \mathrm{L}$ luciferase lysis buffer by injector $\mathrm{M}$ followed by $25 \mu \mathrm{L}$ luciferin solution from Injector P to each well of the luminescent white plate.

5. Monitor luminescence released for $10 \mathrm{~s}$ after luciferin addition. Examples of the data generated by these luciferase reporter assays can be seen in Figs. 1 and 2. 


\subsection{Aequorin Screening}

This screening is primarily made possible upon the coupling of GPCRs to G proteins leading to downstream PLC $\beta$ activation, $\mathrm{IP}_{3}$ accumulation and $\mathrm{Ca}^{2+}$ release from

endoplasmic reticulum. The released $\mathrm{Ca}^{2+}$ is quantified by the presence of $\mathrm{Ca}^{2+}$-sensitive aequorin photoprotein. The aequorin complex is composed of apoaequorin, coelenterazine (a cofactor) and oxygen, which readily reacts with $\mathrm{Ca}^{2+}$ causing the degradation of the coelenterazine bound aequorin, generating coelenteramide and luminescent flashes. Both transient and stable transfections of the apoaequorin have been tested but we find that the response generated from the transient introduction of apoaequorin gives better signal-to-noise ratios.

\subsubsection{Transient transfection (see Note 3)}

1. COS-7 cells are seeded at 10,000 cells/well using DMEM (10\% FBS) in 96-well white luminescent plates.

2. The following day, prepare transfection cocktail as described below:

a. Mix $100 \mathrm{ng}$ apoaequorin cDNA, 50 ng GPCR and G protein cDNAs per well.

b. For each well, dilute $0.2 \mu \mathrm{L}$ of PLUS reagent with $25 \mu \mathrm{L}$ Opti-MEM.

c. Add the PLUS/Opti-MEM diluent to the cDNA mix.

d. Incubate at room temperature for $15 \mathrm{~min}$.

e. Dilute $0.2 \mu \mathrm{L}$ of Lipofectamine reagent with $25 \mu \mathrm{L}$ Opti-MEM per well.

f. Add the diluted Lipofectamine to the cDNA/PLUS/Opti-MEM mix.

g. Incubate at room temperature for a further $15 \mathrm{~min}$. 
3. Remove growth medium from the 96-well microplate.

4. Aliquot $50 \mu \mathrm{l}$ of the transfection cocktail to each well of the microplate.

5. Incubate at $37^{\circ} \mathrm{C}$ for $3 \mathrm{~h}$.

6. Add $50 \mu \mathrm{L}$ Opti-MEM containing 4\% FBS (see Note 7).

7. Incubate at $37^{\circ} \mathrm{C}$ for $48 \mathrm{~h}$.

\subsubsection{Apoaequorin labeling (see Note 8)}

1. For each well prepare labeling medium consisting of $100 \mu \mathrm{L}$ calcium-free HBSS (pH 7.5) with $2.5 \mu \mathrm{M}$ coelenterazine $f$ and $20 \mathrm{mM}$ HEPES (pH 7.4).

2. The transfection medium is removed from the 96-well microplate and replaced by $100 \mu \mathrm{l}$ of the labeling medium.

3. Place the microplate in a dark, humidified incubator at $37^{\circ} \mathrm{C}$ for $4 \mathrm{~h}$.

\subsubsection{Aequorin assay (see Note 9)}

1. The luminometer should be prewarmed to $37^{\circ} \mathrm{C}$.

2. Prepare appropriate controls and drug solutions in $3 \mathrm{X}$ concentrations (50 $\mu \mathrm{l}$ per well will be required).

3. Warm the drug solutions to $37^{\circ} \mathrm{C}$.

4. Set Injector P to inject $50 \mu \mathrm{L}$ control or drug solutions.

5. Following injection, monitor the luminescence released for $15 \mathrm{~s}$. Examples of the types of data generated by aequorin assays can be seen in Figs. 3 and 4 .

\subsection{Data Analysis}


WinGlow software is supplied with the luminometer. This software, in addition to recording the experimental parameters used, allows direct data transfer to Microsoft Excel as Macros for analysis. Data is exported as a time course (both before and after the stimulus injection) of the relative luminescent unit (RLU) released. The readouts in Excel include the peak value, the time when the peak value is reached and the integrated area under the curve in terms of RLU. For both luciferase and aequorin assays, to ensure better reproducibility within assays, we prefer to rely on integrated areas beneath the curves, i.e. $10 \mathrm{~s}$ for luciferase or $15 \mathrm{~s}$ for aequorin assays. To further improve the consistency between assays performed on different days, other standards are useful. The first common standard used is the introduction and normalization of the response to an empty vector control. In other cases, for instance, when the efficacy of an agonist is to be evaluated, the fold of stimulation or percent of stimulation over a blank control is used.

\section{Notes}

1. Transfections may be done in duplicate. Plates should also be seeded for mock transfections (i.e. cells are transfected in the absence of cDNA) to allow for determination of the end point of the selection procedure (also see Note 2). For calcium phosphate transfection of HEK 293 cells, cells should be seeded at approximately $40-60 \%$ confluency, Seeding cells just before the day of transfection avoids crowding of cells that may lower the transfection efficiency. The $\mathrm{pH}$ of the HBS and the incubation time with the transfection cocktail play significant roles in the transfection efficiency. Incubation time periods normally ranges from $8 \mathrm{~h}$ to a maximum of $16 \mathrm{~h}$. Prolonged incubation of cells with the 
calcium phosphate precipitate induces cell death. In addition, after incubation with the transfection cocktail it is crucial to thoroughly rinse the cells with PBS to completely remove the calcium phosphate, however care should be taken not to wash the cells from the plate.

2. Cell death starts to be observable after G418 has been introduced for the first four to five days. Cells should be washed with PBS and new G418 should be replenished into the fresh medium every few days. Normally, the whole selection process takes two to three weeks to finish, at which point all cells in the mock transfected plates should be dead and any living cells in the transfected plates can be assumed to be stably expressing the reporter gene constructs.

3. Triplicates are always performed in our 96-well format assays and often many 96well plates are transfected with the same cDNAs. Hence, instead of mixing cDNAs for individual well, "master mixes" for a number of wells are prepared to reduce the number of pipetting steps and possible pipetting errors. A multichannel pipetman is employed to ease the process of removing growth medium and aliquotting transfection cocktail to microplates. Complete removal of the growth medium before addition of the transfection cocktail is essential as serum in the medium hinders the transfection by LipofectaminePLUS reagents.

4. As phenol red has been shown to have an effect on the luciferin reaction, phenol red-free medium is used for drug treatment after transfection. In addition, the transfection cocktail must be thoroughly removed to avoid activation of the response elements by growth factors, hormones etc in the serum. At least one freeze-thaw cycle is required for complete cell lysis by the luciferase lysis buffer. 
Thus, the collected cell lysates can be kept at $-80^{\circ} \mathrm{C}$ until required for the luciferase assays.

5. For CRE-driven luciferase reporter, two different setups are optimized for the evaluation of either the activation or inhibition of adenylyl cyclase through the regulation of $G_{s}$ or $G_{i / o}$ proteins, respectively. For the activation of $G_{s}$ proteins the experimental setup is similar to that for STAT3- or NFkB-driven luciferase assays. However, for measurements of $\mathrm{G}_{\mathrm{i} / \mathrm{o}}$ protein-mediated activity, the activation of $\mathrm{G}_{\mathrm{i} / \mathrm{o}}$ proteins is revealed through their inhibition adenylyl cyclase activity. Adenylyl cyclase can be activated either directly by forskolin or through the activation of $G_{s}$ proteins by a $G_{s}$-coupled GPCR. In most of our studies, forskolin is used as it guarantees robust stimulation of adenylyl cyclase. This necessitates the use of extra controls. Generally, we use basal, agonist for the receptor under investigation, forskolin alone and forskolin with receptor's agonist. The washing step is particularly important in CRE-driven luciferase assays using forskolin. Traces of forskolin induce remarkable CRE-driven luciferase activity and overwhelm the inhibitory effects mediated by the $\mathrm{G}_{\mathrm{i} / \mathrm{o}}$ proteins. Complete removal of residual forskolin is accomplished by careful aspiration of stimuli and additional washing steps with plain medium. Alternatively, the activation of a $\mathrm{G}_{\mathrm{s}^{-}}$ mediated pathway can be achieved by co-transfection of a $\mathrm{G}_{\mathrm{s}}$-coupled receptor. This $\mathrm{G}_{\mathrm{s}}$-mediated activation of adenylyl cyclase in general is weaker than the forskolin-mediated stimulation, and may be preferred in the examination of $\mathrm{G}_{\mathrm{i}^{-}}$ coupled receptors that couple weakly to adenylyl cyclase. 
6. To maintain consistency in between runs, the luciferin substrate solution, the lysis buffer and the microplate reader are always prewarmed to ambient temperature before assays. An additional $25 \mu \mathrm{L}$ lysis buffer is injected to ensure that the samples of cell lysates are equilibrated to ambient temperature and the cell lysate is evenly mixed and distributed throughout the well.

7. The growth rate for COS-7 cells is faster than HEK 293 cells; thus, a final concentration of $2 \%$ fetal bovine serum is used for the replenishment after transfection.

8. Activated aequorin, i.e. coelenterazin $f$-bound apoaequorin, reacts quickly with $\mathrm{Ca}^{2+}$ for luminescence release. Therefore, it is absolutely essential to maintain a $\mathrm{Ca}^{2+}$-free environment starting from the labeling step.

9. Since intact cells are used for the aequorin assays, the residing platform inside the luminometer, the microplate and the plastic tubes with the drug solutions are warmed to $37^{\circ} \mathrm{C}$. A sudden temperature change for charged cells could cause stress and induce inaccurate readings. Since only one injector (Injector P) is available and suitable in MicroLumat (Berthold) for drug distribution, the screening itself always starts with wells for the basal followed by wells with agonists: otherwise, washings steps are necessary to completely flush out ligands left in the injector tubing.

\section{Acknowledgements}

This work was supported in part by the Hong Kong Jockey Club and grants from the Research Grants Council of Hong Kong (HKUST 6420/05M) and the University Grants 
Committee (AoE/B-15/01). YHW was a recipient of the Croucher Senior Research Fellowship. 


\section{References}

1. Ponglikitmongkol, M., White, J. and Chambon, P. (1990) Synergistic activation of transcription by the human estrogen receptor bound to tandem responsive elements. EMBO J. 9, 2221--2231.

2. Miller-Martini, D.M., Chan, R.Y.K., Ip, N.Y., Sheu, S.J. and Wong, Y.H. (2001) A reporter gene assay for the detection of phytoestrogens in traditional Chinese medicine. Phytotherapy Res. 15, 487--492.

3. New, D.C., Miller-Martini, D.M. and Wong, Y.H. (2003) Reporter gene assays and their applications to bioassays of natural products. Phytotherapy Res. 17, 439--448.

4. Kent, T.C., Thompson, K.S. and Naylor, L.H. (2005) Development of a generic dualreporter gene assay for screening G-protein-coupled receptors. J. Biomol. Screen. 10, 437--446.

5. Hanson, B.J. (2006) Multiplexing Fluo-4 NW and a GeneBLAzer transcriptional assay for high-throughput screening of G-protein-coupled receptors. J. Biomol. Screen. 11, 644--651.

6. Do, E.U., Piao, L.Z., Choi, G., Choi, Y.B., Kang, T.M., Shin, J., Chang, Y.J., Nam, H.Y., Kim, H.J. and Kim, S.I. (2006) The high throughput screening of neuropeptide FF2 receptor ligands from Korean herbal plant extracts. Peptides. 27, 997--1004.

7. Lo, R.K. and Wong, Y.H. (2004) Signal transducer and activator of transcription 3 activation by the $\delta$-opioid receptor via $\mathrm{G \alpha}_{14}$ involves multiple intermediates. Mol. Pharmacol. 65, 1427--1439.

8. Liu, A.M. and Wong, Y.H. (2005) Activation of nuclear factor $\kappa$ B by somatostatin type 2 receptor in pancreatic acinar AR42J cells involves $G \alpha_{14}$ and multiple signaling 
components: a mechanism requiring protein kinase C, calmodulin-dependent kinase II, ERK, and c-Src. J. Biol. Chem. 280, 34617--34625.

9. Lin, P. and Ye, R.D. (2003) The lysophospholipid receptor G2A activates a specific combination of G proteins and promotes apoptosis. J. Biol. Chem. 278, 14379-14386.

10. Mody, S.M., Joshi, S.A., Ho, M.K.C. and Wong, Y.H. (2000) Incorporation of $\mathrm{G \alpha}_{z^{-}}$ specific sequence at the carboxy terminus increases the promiscuity of $\mathrm{G}_{16}$ towards Gi-coupled receptors. Mol. Pharmacol. 57, 13--23.

11. New, D.C. and Wong, Y.H. (2004) Characterization of CHO cell lines stably expressing a $\mathrm{G \alpha}_{16 / \mathrm{z}}$ chimera for high throughput screening of GPCRs. Assay and Drug Develop. Technol. 2, 269--280.

12. New, D.C. and Wong, Y.H. (2005) Chimeric and promiscuous G proteins in drug discovery and the deorphanization of GPCRs. Drug Design Reviews-Online. 2, 66-79.

13. Liu, A.M.F., Ho, M.K.C., Wong, C.S.S., Chan, J.H.P., Pau, A.H.M. and Wong, Y.H. (2003) $\mathrm{G} \alpha_{16 / z}$ chimeras efficiently link a wide range of $G$ protein-coupled receptors to calcium mobilization. J. Biomol. Screen. 8, 39--49.

14. Ungrin, M., Singh, L., Stocco, R., Sas, D. and Abramovitz, M (1999) An automated aequorin luminescent-based functional calcium assay for GPCRs. Anal. Biochem. 272, 34--42.

15. Le Poul, E., Hisada, S., Mizuguchi, Y., Dupriez, V.J., Burgeon, E. and Detheux, M. (2002) Adaptation of aequorin functional assay to high throughput screening. $J$. Biomol. Screen. 7, 57--65. 


\section{Figure Legends}

Figure 1. Dose response curve of $\mathbf{N}^{6}$-cyclohexyladenosine (CHA, selective agonist for adenosine $A_{1}$ receptor) for $A_{1} R / G \alpha_{16}$-transfected $H E K 293$ cells stably carrying a NFkB-driven luciferase reporter construct. To eliminate $G_{i / o}$ signal transduction, transfectants were pre-treated with pertussis toxin $(100 \mathrm{ng} / \mathrm{ml})$ for $4 \mathrm{~h}$ prior to agonist induction. Different concentrations of CHA were added to the cells for $16 \mathrm{~h}$ and the luciferase activities of the cell lysates were measured. (A) Triplicates were performed and the results from one set of wells are illustrated as representative. (B) Construction of the dose response curve was accomplished by plotting the \% stimulation corrected to the basal versus the logarithmic concentrations of CHA. Data shown represent the mean \pm S.E. performed in triplicates.

Figure 2. The inhibitory responses of melatonin type 1 and type 2 receptors (MT and $\mathrm{MT}_{2}$ ) coupled to $\mathrm{G}_{\mathrm{i} / \mathrm{o}}$ proteins as measured by a $\mathrm{CRE}$-driven reporter gene. HEK 293 cells stably carrying a CRE-driven luciferase reporter construct were transfected with $\mathrm{MT}_{1}$ or $\mathrm{MT}_{2}$ and the transfectants were challenged with plain medium (basal), 2iodomelatonin (an agonist at $\mathrm{MT}_{1}$ and $\mathrm{MT}_{2}$; 2-IMT, $10 \mu \mathrm{M}$ ), forskolin (FSK, $10 \mu \mathrm{M}$ ) or FSK with 2-IMT. (A) Raw integrated RLUs were plotted and data shown represent the mean \pm S.E. performed in triplicates. (B) A panel of 2-IMT derivatives was exposed to $\mathrm{MT}_{1}$-transfected HEK 293 cells stably carrying the CRE-driven luciferase reporter. The magnitudes of \% inhibition of the forskolin response (where FSK stimulation alone was set to $100 \%$ ) were plotted and data shown represent the mean \pm S.E. performed in triplicates. *, agonist-induced RLU is significantly higher than the basal (Dunnett's $t$ test, 
$p<0.05)$; , agonist-induced response was significantly inhibited as compared to FSK, (Dunnett's $t$ test, $p<0.05$ ).

Figure 3. Dose response curves of carbachol for COS-7 cells transiently transfected with muscarinic type 1 receptor $\left(M_{1} R\right)$ and apoaequorin. Transfectants were labeled with $2.5 \mu \mathrm{M}$ coelenterazine $f$ for $4 \mathrm{~h}$. (A) Serial dilutions of carbachol were prepared and screening was performed in increasing order of carbachol concentrations. Triplicates were performed and the results from one set of wells are illustrated as representative. (B) Construction of dose response curve was accomplished by plotting the total integrated RLUs versus the logarithmic concentrations of carbachol. Data shown represent the mean \pm S.E. performed in triplicates.

Figure 4. Aequorin assay of the effects of $\mathrm{GABA}_{\mathrm{B}}$-selective antagonists in COS-7 cells transfected with $\mathrm{GABA}_{\mathrm{B} 2}$ and either $\mathrm{GABA}_{\mathrm{B} 1 \mathrm{a}}$ or $\mathrm{GABA}_{\mathrm{B} 1 \mathrm{~b}}$ receptor subunits as well as 16z25. Transfectants were treated with basal (open bar), GABA (500 $\mu \mathrm{M}$; closed bar), GABA with 2-hydroxy saclofen (2-OH Sac; $50 \mu \mathrm{M}$; diagonally striped bar) and GABA with saclofen (50 $\mu \mathrm{M}$, grey bar). 2-OH Sac and saclofen are antagonists at $\mathrm{GABA}_{\mathrm{B}}$ receptors and they were added simultaneously with GABA. Raw integrated RLUs and \% stimulation (as compared to their corresponding basals) were plotted. Data shown represent the mean \pm S.E. performed in triplicates. *, agonist-induced RLU is significantly higher than the basal (Dunnett's $t$ test, $p<0.05$ ); \#, antagonist significantly suppressed the GABA-induced $\mathrm{Ca}^{2+}$ mobilization (Dunnett's $t$ test, $p<0.05$ ). 